\title{
Editorial
}

\section{Impacts of Climate Change on Biological Dynamics}

\author{
Gui-Quan Sun, ${ }^{1}$ Xue-Zhi Li, ${ }^{2}$ Yi Wang, ${ }^{3,4}$ Amit Chakraborty, ${ }^{5}$ \\ Zhen Wang, ${ }^{6}$ and Yong-Ping $\mathrm{Wu}^{7,8}$ \\ ${ }^{1}$ Complex Systems Research Center, Shanxi University, Taiyuan, Shanxi 030006, China \\ ${ }^{2}$ Department of Mathematics and Physics, Anyang Institute of Technology, Anyang 455000, China \\ ${ }^{3}$ Department of Mathematics and Statistics, University of Victoria, Victoria, BC, Canada V8W 3R4 \\ ${ }^{4}$ Department of Mathematics, Southeast University, Nanjing 210096, China \\ ${ }^{5}$ Ecological Complexity and Modeling Laboratory, University of California, Riverside, CA 92521-0124, USA \\ ${ }^{6}$ Interdisciplinary Graduate School of Engineering Sciences, Kyushu University, Fukuoka 816-8580, Japan \\ ${ }^{7}$ College of Physics Science and Technology, Yangzhou University, Yangzhou, Jiangsu 225002, China \\ ${ }^{8}$ Department of Botany and Plant Sciences, University of California, Riverside, CA 92521-0124, USA
}

Correspondence should be addressed to Gui-Quan Sun; gquansun@126.com

Received 9 May 2016; Accepted 9 May 2016

Copyright (c) 2016 Gui-Quan Sun et al. This is an open access article distributed under the Creative Commons Attribution License, which permits unrestricted use, distribution, and reproduction in any medium, provided the original work is properly cited.

A crucial challenge for humanity in coming decades is to reduce the adverse effects of climate change that are already being observed in our planet's environment. Growing human populations are demanding significant increase in economic development that accelerates climate change. Moreover, there is a clear need to substantially reduce many negative effects of industry and agriculture that decrease the adverse effects of climate change. The problem is deepening further with the increasing competition that modern human societies are experiencing for land, water, and energy. Consequences of climate change have several multifaceted effects which might lead to collapse of ecosystems, biological invasion, and loss of biodiversity. To provide new insights on protection, control over the populations of ecosystems and systematic researches on the characteristic of climate change and its impacts on biological dynamics are of great practical significance.

The impacts of climate change on biological dynamics are focused on two aspects. On one hand, more attention should be paid to the characteristics and actual impact of climate change on biological and ecological systems. Researchers need to keep eyes not only on the theoretical findings in the influences of climate change on biological dynamics, but also on the analysis of real data of climate change or other related issues, including diagnosis, evaluation, and challenges to existing areas of effects of climate change on ecosystem dynamics. On the other hand, to adapt to the varied situation caused by climate change, new perspective and methods should be explored and used in study on biodynamic systems. For example, climate change might lead to changes in immune system and immune function of organisms and biological systems, inducing the emergence of new diseases, and some variations of transmission of infectious diseases. Therefore, some previous methods, such as reaction diffusion equations and complex networks, need to be improved.

This special issue received many manuscripts from the scholars in these research fields. The topics of these accepted papers contain descriptions of climate change and ecological systems, analysis on climate and meteorological elements, and some models of disease spread. Some researchers evaluated the influences of climate change, through comparing different data and methods. Others revealed the dynamic behaviors, impact of media, and nonlinear dynamics of diseases.

It is noteworthy that it is impossible to assemble all the works in the fields of the impacts of climate change on biological dynamics. Although more work is needed, this special issue has opened a new start for the explorations on impacts of climate change on biological dynamics. We have good reasons to believe that more outstanding researches on 
this topic will emerge in large numbers. The characteristics of biological systems, modeling biodynamics, and the related adaptation strategies will raise new rush for these fields in the future.

\author{
Gui-Quan Sun \\ Xue-Zhi Li \\ Yi Wang \\ Amit Chakraborty \\ Zhen Wang \\ Yong-Ping Wu
}




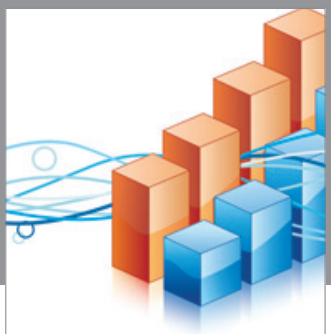

Advances in

Operations Research

vatem alat4

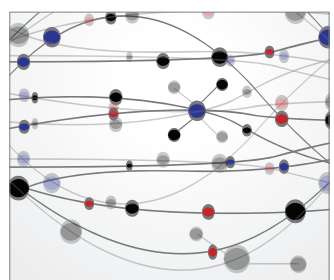

\section{The Scientific} World Journal
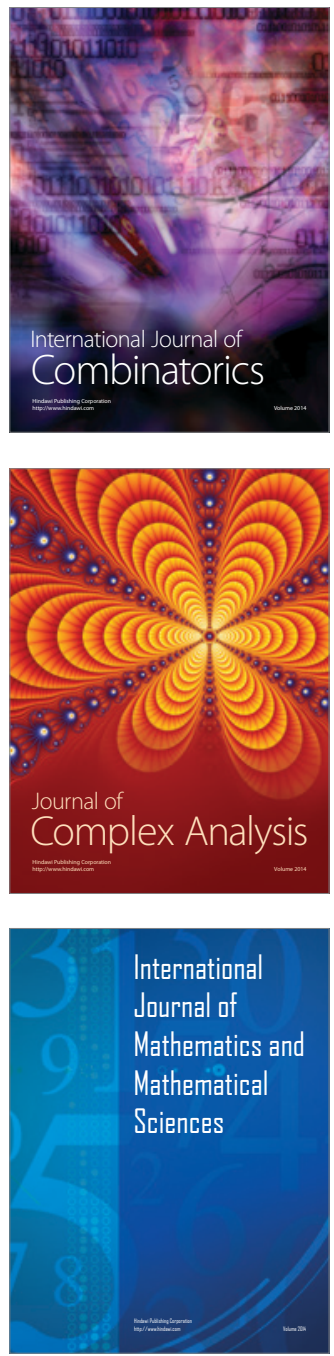
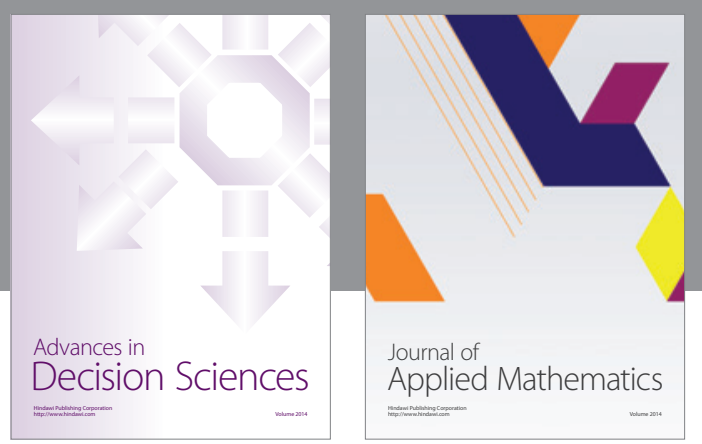

Algebra

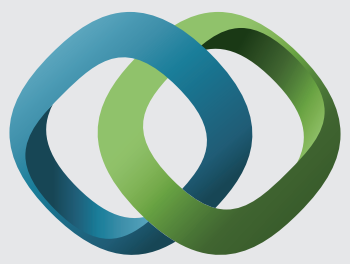

\section{Hindawi}

Submit your manuscripts at

http://www.hindawi.com
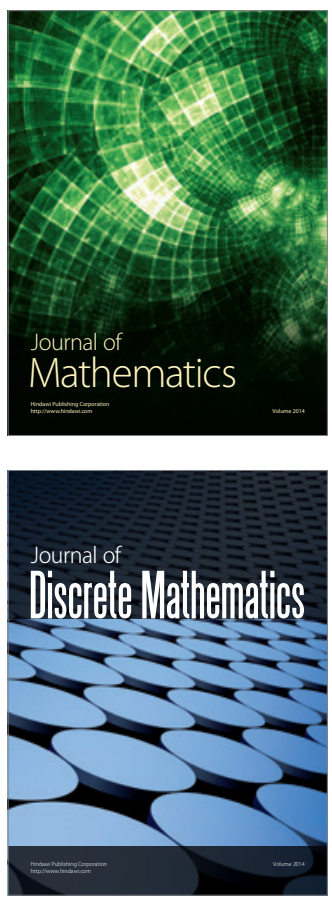

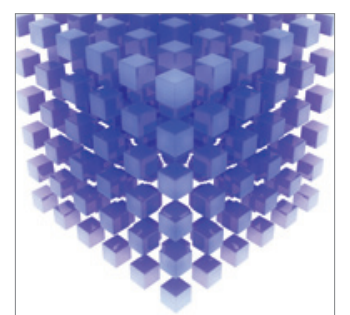

Mathematical Problems in Engineering
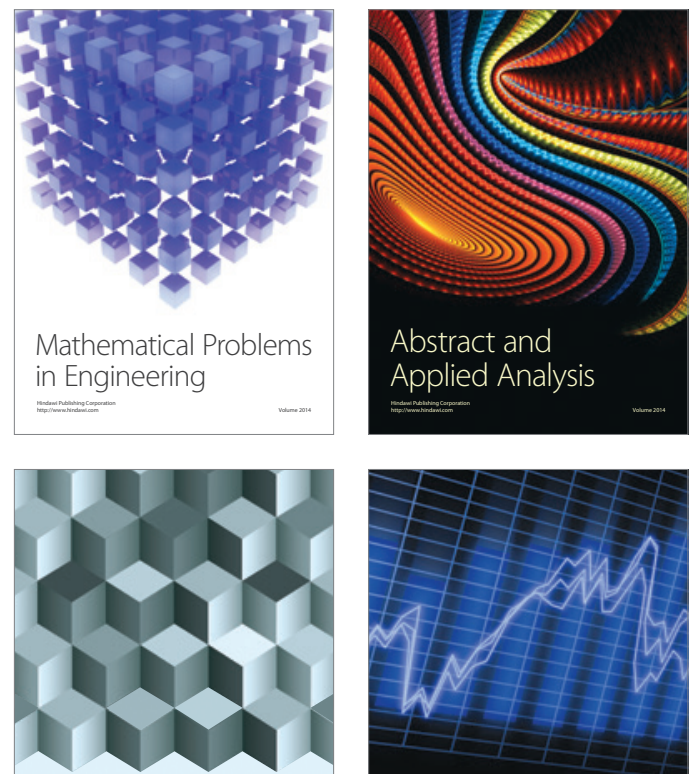

Journal of

Function Spaces

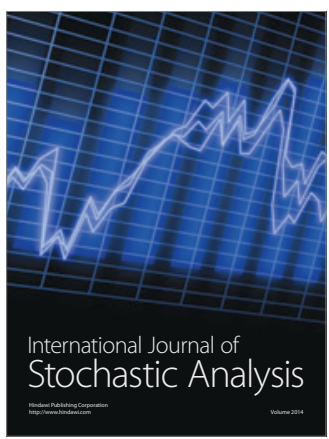

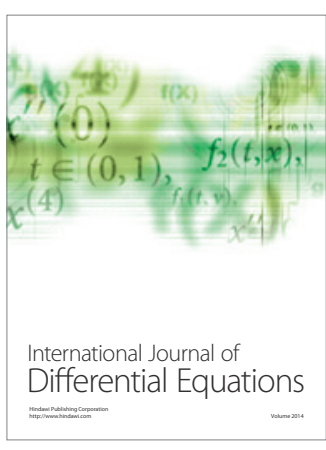
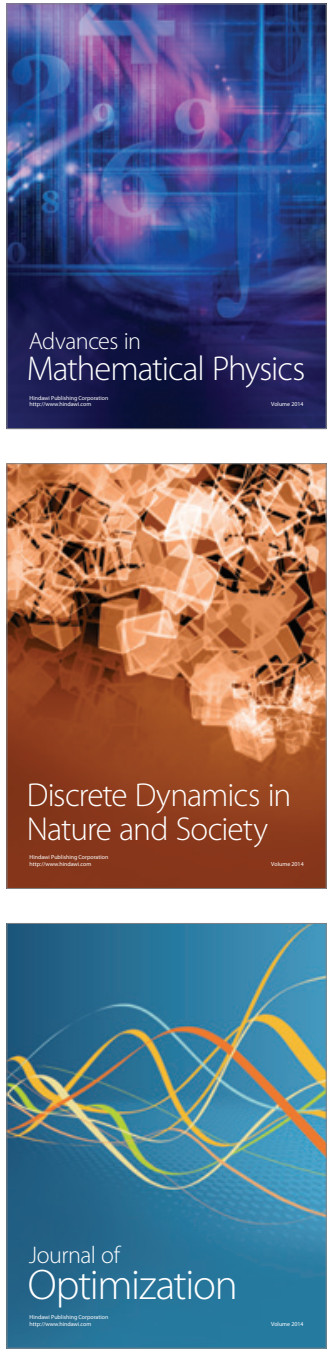